\title{
Speculating on Biodesign in the Future Home
}

\author{
Phillip Gough \\ phillip.gough@sydney.edu.au \\ Design Lab, School of Architecture \\ Design and Planning, The University \\ of Sydney \\ Leigh-Anne Hepburn \\ leigh-anne.hepburn@sydney.edu.au \\ Design Lab, School of Architecture \\ Design and Planning, The University \\ of Sydney

Angela Vujic
avujic@mit.edu
MIT Media Lab
Pattie Maes
pattie@media.mit.edu
MIT Media Lab

\author{
Jack Forman \\ jackform@media.mit.edu \\ MIT Media Lab
}

\author{
Pat Pataranutaporn \\ patpat@mit.edu \\ MIT Media Lab
}

Carolina Ramirez-Figueroa
c.ramirez-figueroa@rca.ac.uk

Royal College of Art, School of Design

David S. Kong

dkong@mit.edu

MIT Media Lab

Hiroshi Ishii

ishii@media.mit.edu

MIT Media Lab

\author{
Naseem Ahmadpour \\ naseem.ahmadpour@sydney.edu.au \\ Affective Interactions Lab, School of \\ Architecture Design and Planning, \\ The University of Sydney
}

\section{CCS CONCEPTS}

- Human-centered computing $\rightarrow$ Interaction design theory, concepts and paradigms; HCI theory, concepts and models; - Applied computing $\rightarrow$ Life and medical sciences.

\section{KEYWORDS}

Biodesign, Speculative Design, Interaction Design, Biological HCI

ACM Reference Format:

Phillip Gough, Jack Forman, Pat Pataranutaporn, Leigh-Anne Hepburn, Carolina Ramirez-Figueroa, Clare Cooper, Angela Vujic, David S. Kong, Raphael Kim, Pattie Maes, Hiroshi Ishii, Misha Sra, and Naseem Ahmadpour. 2021. Speculating on Biodesign in the Future Home. In CHI Conference on Human Factors in Computing Systems Extended Abstracts (CHI '21 Extended Abstracts), May 8-13, 2021, Yokohama, Japan. ACM, New York, NY, USA, 5 pages. https://doi.org/10.1145/3411763.3441353

\section{INTRODUCTION}

Biodesign integrates living organisms into designed solutions, often with the aim of improving sustainability and ecological performance in novel ways [11]. These designs can allow organisms to participate in interactive systems, responding to changes in their own environment in ways that can create interactive systems [17]. Recent research applicable to biodesign analyses biological organisms acting as components within interactive systems [10], and proposes frameworks for biological Human-Computer Interaction (HCI) $[13,14]$. The application of biodesign for $\mathrm{HCI}$ is to combine 
the needs of both the users and the organisms which take part in the system [2]. This presents an exciting opportunity to HCI as it may lead to new innovations for a sustainable future. Biodesign as an approach has been adopted by many, broad fields, including fashion, architecture and art [11]. However, biodesign is becoming more interactive and relevant to HCI, through examples of bio-digital hybrid computational systems [16], shape-changing clothing [17], novel material fabrication [18], grown robots [12], new materials for prototyping interactive systems [7] and public interactive displays [8].

In this workshop, we extend the current narratives in humancentered biodesign to understand the role of interactive biodesign systems at home, as a place with extended functionalities. In doing so, we acknowledge the many definitions of home as a place, space, feeling, practice or an active state of being in the home [9]. We invite participants in the workshop to provide examples of their work within the scope of biodesign, particularly for interactive systems that extend upon or align with these notions.

We acknowledge that when home becomes a place of work, it has implications for the personal, shared and social spaces we use. This also has implications for the way we interact with others and the work tasks we perform. Therefore, creating biodesign systems in this multi-functioning environment will need to address competing human behavior in relation to a few models of interest: (1) proxemics as individual's physical and psychological positioning towards others [3], (2) comfort as satisfaction with a state of physical and psychological harmony [1], (3) wellbeing as a state of optimal functioning due to fulfillment of basic psychological needs such as autonomy, competence and relatedness [15], (4) personal productivity in work and non-work contexts [5] and (5) resilience as the ability to adjust prior to, during, or following change that comes from outside the normally expected boundaries [4].

These models are complex and at times overlapping, raising many important questions, particularly given that augmenting our living and working places with any designed system has implications for fair access (equality and equity) and consequences that raise questions of right or wrong (ethics). We invite workshop participants to share examples of interactive biodesign systems, and discuss the models and questions outlined above. Biodesign has been approached from multiple perspectives, such as HCI, biology, art and ethics,[10]. For this workshop we will employ speculative thinking to explore the potential implications of biodesign in the home before building collaborations that implement new projects [6], and develop a critical understanding of biodesign in everyday life.

\section{BACKGROUND}

This workshop is part of a series of workshops on speculating the future of biodesign in HCI. The first workshop was held as part of ACM DIS2020 conference titled "The Nature of Biodesigned Systems: Directions for HCI" [2]. We examined a key question in biodesign "what will interactive systems look like in a guided and grown environment, rather than a built environment?" In that workshop, we explored technologies that rely on symbiotic relationships between the user and organisms that participate in interactive systems. Through exploring new aspects of designing for living computational systems in the ACM DIS2020 workshop, we identified three research themes and questions related to (1) the need for system thinking to consider biodesign ecologies and their complex inter-relationship and impact on human life (rather than just one component or product); (2) identifying stakeholders who lead biodesign research in different contexts, and; (3) questions of values and ethics.

We organised the DIS2020 workshop remotely with participants joining us from their homes across the globe, many in lock-down due to the COVID-19 pandemic. We discussed not only their work in biodesign but also the challenges they faced in their practice. It was clear that home as a research and innovation site will form an important part of future investigations. We therefore decided to examine the three themes from DIS2020 workshop in the context of home in CHI2021.

\section{KEY QUESTIONS}

In this workshop we explore how previous work may be extended as home and work places overlap. The outcomes will deepen our understanding of interactive biodesign systems with meanings attributed to home and work.

We invite participants to explore the following themes and questions with us:

(1) understanding the context: how may the future home be different, due to global challenges and advances in technology?

(2) opportunities for intervention: can biodesign be a nonspeculative intervention in this context, and with the current state of biodesign?

(3) speculating on future interventions: What do they look like? What will they accomplish? How can speculative interventions be mapped to coexist and relate to each other?

\subsection{Website}

All communications about the workshop will be through the workshop website at:

http://biopolisproject.com/chi2021-biodesign/

A copy of the call for position papers will be posted with updated details about workshop activities. All accepted position papers will be also announced and posted on the workshop website.

\section{ORGANISERS}

Phillip Gough, PhD. is a Lecturer in biological design at the University of Sydney School of Architecture, Design and Planning and Program Director for the Major in Biological Design. His teaching and research focus on interdisciplinary collaboration between design and science, including biodesign, non-expert user data visualisation, and design for health and wellbeing.

fack Forman. is a graduate student at the MIT Media Lab, in the Tangible Media Group. Jack's vision is to make tangible artifacts embedded seamlessly with responsive behavior, through the development of programmable materials and ways to fabricate them, to make engaging human-material interfaces that utilize the emotional power of touch. Jack sees a future where these adaptive interfaces are ubiquitous, where every material is able to accept and respond to the change that is constantly flowing through us. Previously, 
Jack received his B.S. from Carnegie Mellon University, where he double majored in Materials Science and Biomedical Engineering.

Pat Pataranutaporn. is a technologist, designer, and a graduate student at the MIT Media Lab. Pat's interests are at the intersection of biotechnology and wearable technology, specifically at the integration of bio and digital system to create personalized interfaces for the users. In his thesis, Pat developed Biological HCI, a design framework that investigates the relationships between human, computer and biological systems by defining biological materials as design elements.

Leigh-Anne Hepburn, PhD. is a senior lecturer at the Design Lab, The University of Sydney. Her research utilises participatory and social innovation at the intersections of industry, academia, government and community to co-design new models of resilience and transdisciplinary collaboration.

Carolina Ramirez-Figueroa, PhD. is a Research Tutor in the Design Products programme at the Royal College of Art, and visiting Lecturer at the Bartlett School of Architecture UCL. Her work combines critical theory, feminist studies and creative practice to look at the intersection of architecture, design and living systems.

Clare Cooper, $P h D$. is a Lecturer at the University of Sydney. Her research and practice span design futuring, workshop facilitation, performance and design activism. Over the last two decades, Cooper has brought together thousands of people to work collectively on community initiatives, creative approaches to governance, collaborative composition, speculative design and critical listening through co-founding the NOW now festival (2001), The Splinter Orchestra (2001), Berlin Splitter Orchester (2009) and Frontyard Projects (2016).

Angela Vujic. is a graduate researcher at the Fluid Interfaces Group in the MIT Media Lab. She is motivated by the connection between the microbiome and mental health to create technology that mediates the mind-gut connection. She aims to introduce gut-brain computer interfaces (GBCIs) to HCI, devices that enable individuals to modulate their gut activity or use their gut activity as inputs to other devices. She holds a degree in computer science from Georgia Tech where she developed brain-computer interface (BCI) glasses with a fiber optic display for assistive communication.

Raphael Kim, PhD. is a designer and HCI researcher, investigating our fraught, and often peculiar relationships with biology and technology. He designs playful artefacts, which incorporate living, (micro-)biological materials with computer systems, to better understand the implications of our bio-digitally mediated futures. Following his master's degree and visiting lectureship at Design Interactions, Royal College of Art (RCA), Raphael gained his Ph.D degree in 2020 from Media and Arts Technology program, Queen Mary University in London, UK.

David Sun Kong, PhD. is a Synthetic Biologist, community organizer, musician, and photographer based in Lexington, MA. He is the Director of the MIT Media Lab's new Community Biotechnology Initiative. He was recognized as an emerging leader in synthetic biology as a "LEAP" fellow, served as a guest faculty member at the Marine Biology Lab in Woods Hole, MA, and is co-founder and managing faculty of "How To Grow (Almost) Anything," an international course on synthetic biology.

Hiroshi Ishii, PhD. Hiroshi Ishii is the Jerome B. Wiesner Professor of Media Arts and Sciences at the MIT Media Lab. After joining the Lab in October 1995, he founded the Tangible Media Group to make digital tangible by giving physical form to digital information and computation. Here, he pursues his visions of Tangible Bits (1997) and Radical Atoms (2012) that will transcend the Painted Bits of GUIs (Graphical User Interfaces), the current dominant paradigm of HCI (Human-Computer Interaction). He received the 2019 SIGCHI Lifetime Research Award.

Pattie Maes, $P h D$. is a professor in MIT's Program in Media Arts and Sciences and runs the Media Lab's Fluid Interfaces research group, which aims to radically reinvent the human-machine experience. Coming from a background in artificial intelligence and human-computer interaction, she is particularly interested in the topic of cognitive augmentation, or how immersive and wearable systems can actively assist people with memory, learning, decision making, communication, and wellbeing.

Misha Sra, PhD. is the John and Eileen Gerngross Assistant Professor and directs the Perceptual Engineering Lab in the Computer Science department at UCSB. She is also affiliated with UCSB's Center for Responsible Machine Learning (CRML). Misha received her PhD at the MIT Media Lab in 2018. She has published at the most selective HCI and VR venues such as CHI, UIST, VRST, and DIS where she received multiple best paper awards and honorable mentions.

Naseem Ahmadpour, PhD. is Senior Lecturer and the director of Affective Interactions lab, The University of Sydney. She conducts research at the cross-section of HCI, design, and psychology, and regularly publishes in HCI journals and conferences. She is interested in researching emerging technologies that support human flourishing through reflection and affective experiences.

\section{WORKSHOP PLAN}

\subsection{Before the Workshop}

Participants are expected to submit a short position papers (1-3 pages, in ACM extended abstract format). The position papers are submitted through the workshop website. The position papers may provide contributions and critical thinking perspectives aligned with the workshop theme. Research on interactive biodesign systems is a growing area of research and innovation and we invite participants to share their thoughts, reflections and experiences in this area relevant to home and work. Submissions will be juried by the organizers based on relevance.

\subsection{Workshop Activities}

This two-day workshop will provide a unique combination of activities to generate insight into upcoming trends in researching and creating interactive biodesign systems at home. The workshop will be held online via Zoom. Both workshop sessions will last 3 hours. Workshop activities will be facilitated through the Miro online collaboration tool. 
Day 1: Following a short introduction, participants are invited to to present their position papers in Pecha Kucha style (using either 20x20-second slides or 5x1-minutes slides). Participants will then engage in an activity to work collaboratively and create a visual map of biodesign technology topics in given presentations (on Miro). Next, participants will use post-it notes on Miro to identify the opportunities and roadmaps for these trends to change the future of home and work, noting the potential consequences and impacts on individuals, families, communities and society. Finally, participants receive directions for a brief overnight observational activity. In this activity, participant will pick an everyday situation, object or device at home and speculate a "what if..." scenario. This involves re-framing the situation, object or device using interactive biodesign properties, aligned with the trends identified earlier. Each participant will be able to share their scenario (in picture or writing) using a template provided on Miro.

Day 2: In day two, participants will report on their overnight activity and present their speculated scenarios on Miro. Next, participants will imagine a biodesign ecology using speculated scenarios, using a virtual card deck to facilitate this activity. Participants will be able to contribute using virtual tools. We are interested in biodesign ecologies as it was discussed extensively in our previous workshop at ACM DIS2020. In this workshop we explore how such ecology might look, and its impact our future homes. Our team is experienced in organising this type of speculative workshop. We are researchers and educators in biodesign and HCI with expertise in co-design and futuring. Day two ends with a reflective discussion on the ethical ramifications of the speculated future and identifying directions for further research and innovation.

\section{Day 1}

- Introduction (15 minutes)

- Project Presentations

- Break (15 minutes)

- Activity 1: creating a visual map of current research and practice trends (1 hour)

- Overnight Activity (15 minutes)

\section{Day 2}

- Reporting on overnight activity (30 minutes)

- Activity 2: Speculating a biodesign ecology (1 hour)

- Break (15 minutes)

- Final reflection (30 minutes)

- Close (15 minutes)

\section{POST-WORKSHOP PLAN}

The workshop will bring together a community of researchers and practitioners interested in biodesign, interactive HCI systems, speculative methods and future of home and work. We aim to build new collaborations through this workshop. Findings and a summary of opportunities identified for this field will be communicated through subsequent publications and participants will be invited to contribute to that. A summary of the discussions will be posted on the workshop website.

\section{CALL FOR PARTICIPATION}

This two day workshop will bring together participants to build a community around biodesign and HCI, broadly defined. We invite participants from research, practice and industry, who are interested in speculative futures of interactive biodesign systems, to participate in our workshop. We are interested in hearing diverse voices of those who combine knowledge in areas of biological science, HCI, speculative and design fiction.

Participants are invited to submit a short position paper (2-4 pages long, excluding references) examining the future of home and work through: literature review, case study review, research protocols, reports of work-in-progress, ethical consequences or relevant methods and tools. Position papers should be in the ACM SIGCHI Master Article template. Submissions will be juried by the organisers based on relevance to the workshop theme. Submissions and questions should be submitted via the workshop website. At least one author of each accepted paper must attend the workshop.

Participants will have the opportunity to present their proposal in the workshop, receive feedback and engage in discussion and a range of speculative activities around the opportunities for biodesign in the future home. The outcomes will broaden our critical understanding of this emerging field as well as ways through which we can approach biodesign innovations in the future.

Key dates

- Call for participation (10 December)

- Position papers (21 February)

- Notification of acceptance (28 February)

\section{REFERENCES}

[1] Naseem Ahmadpour. 2017. Comfort experience in everyday life events. In Advances in Affective and Pleasurable Design. Springer, 625-632.

[2] Phillip Gough, Larissa Pschetz, Naseem Ahmadpour, Leigh-Anne Hepburn, Clare Cooper, Carolina Ramirez-Figueroa, and Oron Catts. 2020. The Nature of biodesigned systems: Directions for HCI. In Companion Publication of the 2020 ACM Designing Interactive Systems Conference. 389-392.

[3] Edward Twitchell Hall. 1966. The hidden dimension. Vol. 609. Garden City, NY: Doubleday.

[4] Erik Hollnagel, David D Woods, and Nancy Leveson. 2006. Resilience engineering: Concepts and precepts. Ashgate Publishing, Ltd.

[5] Young-Ho Kim, Eun Kyoung Choe, Bongshin Lee, and Jinwook Seo. 2019. Understanding personal productivity: How knowledge workers define, evaluate, and reflect on their productivity. In Proceedings of the 2019 CHI Conference on Human Factors in Computing Systems. 1-12.

[6] Shaun Lawson, Ben Kirman, Conor Linehan, Tom Feltwell, and Lisa Hopkins. 2015. Problematising Upstream Technology through Speculative Design. In Proceedings of the 33rd Annual ACM Conference on Human Factors in Computing Systems - CHI '15, Vol. 2015-April. ACM Press, New York, New York, USA, 2663-2672. https://doi.org/10.1145/2702123.2702260

[7] Eldy S. Lazaro Vasquez and Katia Vega. 2019. Demo: From plastic to biomaterials: Prototyping DIY electronics with mycelium. UbiComp/ISWC 2019- - Adjunct Proceedings of the 2019 ACM International foint Conference on Pervasive and Ubiquitous Computing and Proceedings of the 2019 ACM International Symposium on Wearable Computers (2019), 308-311. https://doi.org/10.1145/3341162.3343808

[8] Kyungwon Lee, Jaewoo Jung, and Seung Ah Lee. 2020. MicroAquarium: An immersive and interactive installation with living microorganisms. Conference on Human Factors in Computing Systems - Proceedings (2020), 1-4. https://doi. org $/ 10.1145 / 3334480.3383164$

[9] Shelley Mallett. 2004. Understanding home: a critical review of the literature. The sociological review 52, 1 (2004), 62-89.

[10] Timothy Merritt, Foad Hamidi, Mirela Alistar, and Marta DeMenezes. 2020. Living media interfaces: a multi-perspective analysis of biological materials for interaction. Digital Creativity 31, 1 (jan 2020), 1-21. https://doi.org/10.1080/ 14626268.2019.1707231

[11] William Myers. 2012. Bio design. Museum of Modern Art.

[12] Pat Pataranutaporn, Jaime Sanchez De La Vega, Abhik Chowdhury, Audrey Ng, and Galina Mihaleva. 2018. Toward Growable Robot : Exploring and Integrating Flexible - Biological Matter with Electronics. In 2018 International Flexible Electronics Technology Conference (IFETC). IEEE, 1-4. https://doi.org/10.1109/IFETC. 2018.8584034

[13] Pat Pataranutaporn, Todd Ingalls, and Ed Finn. 2018. Biological HCI: towards integrative interfaces between people, computer, and biological materials. In 
Extended Abstracts of the 2018 CHI Conference on Human Factors in Computing Systems. 1-6.

[14] Pat Pataranutaporn, Angela Vujic, David S. Kong, Pattie Maes, and Misha Sra 2020. Living Bits. In Proceedings of the Augmented Humans International Conference. ACM, New York, NY, USA, 1-12. https://doi.org/10.1145/3384657.3384783

[15] Dorian Peters, Naseem Ahmadpour, and Rafael A Calvo. 2020. Tools for WellbeingSupportive Design: Features, Characteristics, and Prototypes. Multimodal Tech nologies and Interaction 4, 3 (2020), 40.

[16] Harpreet Sareen and Pattie Maes. 2019. Cyborg botany: Exploring in-planta cybernetic systems for interaction. Conference on Human Factors in Computing
Systems - Proceedings (2019), 1-6. https://doi.org/10.1145/3290607.3313091

[17] Lining Yao, Jifei Ou, Chin-Yi Cheng, Helene Steiner, Wen Wang, Guanyun Wang, and Hiroshi Ishii. 2015. bioLogic. In Proceedings of the 33rd Annual ACM Conference on Human Factors in Computing Systems - CHI '15 (CHI '15). ACM Press, New York, New York, USA, 1-10. https://doi.org/10.1145/2702123.2702611

[18] Jiwei Zhou, Bahareh Barati, Jun Wu, Diana Scherer, and Elvin Karana. 2020. Digital biofabrication to realize the potentials of plant roots for product design. Bio-Design and Manufacturing 0123456789 (sep 2020). https://doi.org/10.1007/ s42242-020-00088-2 